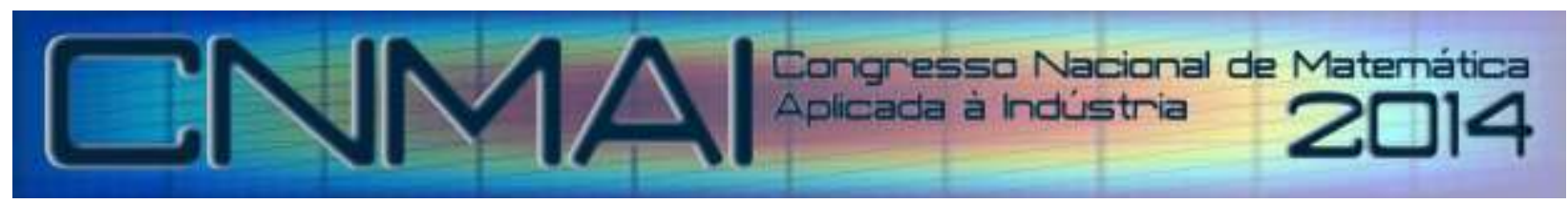

18 a 21 de novembro de 2014, Caldas Novas - Goiás

\title{
TRANSFORMADA DA INCERTEZA PURAMENTE NUMÉRICA PARA O MAPEAMENTO DE DISTRIBUIÇÕES DE PROBABILIDADE
}

\author{
Leonardo da Cunha Brito, brito@emc.ufg.br ${ }^{1}$ \\ Leonardo Rodrigues Araújo Xavier de Menezes, leonardo@ene.unb.br² \\ Adson Silva Rocha, adson.rocha@ifgoiano.edu.br ${ }^{3}$ \\ Ademir Alves de Brito Junior, ademir_britojr@hotmail.com ${ }^{1}$ \\ ${ }^{1}$ Universidade Federal de Goiás - Escola de Engenharia Elétrica, Mecânica e de Computaão, Av. Universitária, n. ${ }^{\circ}$ \\ 1488, qd. 86, bloco A, $3^{\circ}$ piso, Setor Leste Universitário, CEP 74605-010, Goiânia/GO \\ ${ }^{2}$ Universidade de Brasília - Departamento de Engenharia Elétrica, CEP 70900-900, Brasília/DF \\ ${ }^{3}$ Instituto Federal Goiano - Rua 88, n 310, Setor Sul, CEP 74085-010, Goiânia/GO
}

\begin{abstract}
Resumo: Neste trabalho, foi desenvolvida uma versão numérica da Transformada da Incerteza (expressão utilizada para denominar a Unscented Transform, cuja tradução livre é Transformada "Sem cheiro"). Na abordagem desenvolvida, quaisquer distribuições de probabilidade podem ser mapeadas por meio de funções lineares ou nãolineares, permitindo assim a obtenção ágil das distribuições de probabilidade das saídas/respostas do modelo de simulação ou, mais especificamente, do modelo de avaliação de incertezas. Um grau de precisão adequado pode ser atingido, visto que o equacionamento permite o incremento arbitrário de pontos amostrais das funções de mapeamento, em detrimento do aumento do custo computacional demandado. Não obstante, para propósitos práticos de mapeamento de distribuições, o custo computacional ainda se mostra consideravelmente menor que aquele demandado, por exemplo, pelo método de Monte Carlo, o qual é baseado em amostragem aleatória massiva, apresentando assim altíssimo custo computacional. As aplicações em problemas de avaliação de incertezas em Engenharia mostram a aplicabilidade e a eficiência do método proposto.
\end{abstract}

Palavras-chave: mapeamento, distribuição de probabilidade, Transformada da Incerteza, versão numérica

\section{INTRODUÇÃ̃O}

Os valores dos parâmetros de um modelo que mapeia variáveis de entrada para as correspondentes saídas, ou respostas, podem ser expressos deterministicamente, adotando-se um único valor fixo, um valor médio por exemplo, ou podem ser apresentados de forma estatística, caracterizadas por distribuições de probabilidade (Cacuci, 2003). No primeiro caso, apenas uma avaliação para se encontrar a relação entrada-saída é necessária, caso o próprio modelo seja determinístico. No segundo caso, uma quantidade considerável de avaliações da função de mapeamento entrada-saída é demandada. Geralmente, para se ter uma precisão adequada, é necessário um montante de dezenas ou centenas de milhares de execuções, de forma que, dadas as distribuições de probabilidade das variáveis de entrada, as distribuições de probabilidade das variáveis de saída do modelo possam ser estimadas. Há casos para os quais uma quantidade massiva de avaliações não tem um alto custo computacional, ao passo que, para outros, o custo de avaliação é proibitivo, quando uma quantidade considerável delas é necessária. Sendo assim, o desenvolvimento de métodos que proveem uma estimativa precisa, porém com menor esforço computacional, é imperativa nesses casos. A Transformada da Incerteza (Borges et al., 2008; Ihihara et al., 2013; Julier et al., 2004; Menezes et al., 2007; Menezes et al., 2010; Paredes et al., 2011; Paredes et al., 2010; Santos et al., 2009; Thomas et al., 2011), caracterizada pela discretização das distribuições contínuas das variáveis de entrada, associada a aproximações polinomiais aplicadas às funções de mapeamento entradasaída, se apresenta como uma ferramenta matemática de grande valia para este objetivo. 


\section{A TRANSFORMADA DA INCERTEZA PURAMENTE NUMÉRICA}

Uma das dificuldades para a aplicação expedita da Transformada da Incerteza é dada pela necessidade do desenvolvimento algébrico e da solução analítica das equações que compõem o processo de mapeamento das distribuições de entrada e de saída do modelo (Borges et al., 2008). O que é complexo para apenas uma variável de entrada e apenas uma função de mapeamento e sua respectiva saída, torna-se ainda mais complexo quando várias variáveis probabilísticas de entrada e de saída são consideradas, o que pode dificultar consideravelmente ou mesmo inviabilizar a aplicação ágil da Transformada da Incerteza. De forma a suplantar este problema, o presente trabalho apresenta uma metodologia para a solução numérica do processo de transformação. Considera-se, assim, um número arbitrário de variáveis de entrada e de saída e suas distribuições correspondentes pré-definidas, uma precisão de mapeamento arbitrária, associada ao número de pontos sigma fornecido a priori, e um número qualquer de variáveis de saída, cujos momentos e distribuições são estimados a partir da solução numérica das equações.

A Eq. (3) apresenta a equação básica que relaciona o domínio contínuo com o domínio discreto das variáveis probabilísticas de entrada (Menezes et al., 2007), $x=\left({ }^{1} x,{ }^{2} x, \cdots,{ }^{n_{v}} x\right)$. Doravante, esta será denominada equação de equivalência. $\mathrm{O}$ termo ${ }^{r} x_{q}$ representa a $q$-ésima coordenada sigma da $r$-ésima variável aleatória. Assume-se que cada uma das $n_{v}$ variáveis aleatórias de entrada é representada por $n_{s}$ coordenadas sigma. A combinação de todas as coordenadas de todas as variáveis aleatórias formarão os pontos sigma no espaço das variáveis de entrada. Ou seja, temse um total de $N_{s}=n_{s}{ }^{n_{v}}$ pontos sigma, bem como a mesma quantidade de pesos sigma. O peso $w_{j_{1}, j_{2}, \cdots, j_{n_{v}}}$ é referente ao ponto sigma em $\left({ }^{1} x_{j_{1}},{ }^{2} x_{j_{2}}, \cdots,{ }^{n_{v}} x_{j_{v}}\right)$. O número de incógnitas do conjunto de equações é, então, dado por $N_{I}=N_{S}+$ $N_{c}$, na qual $N_{s}$ é o número de pesos e $N_{c}$ é o número total de coordenadas sigma, dado por $N_{c}=n_{s} \times n_{v}$.

$$
\begin{aligned}
\sum_{j_{1}=1}^{n_{s}} \sum_{j_{2}=1}^{n_{s}} \cdots \sum_{j_{n_{v}=1}}^{n_{s}} w_{j_{1}, j_{2}, \cdots, j_{n_{v}}} \times\left({ }^{1} x_{j_{1}}{ }^{1} p_{k} \times{ }^{2} x_{j_{2}}{ }^{2}{ }^{p_{k}} \times \cdots \times{ }^{n_{v}} x_{j_{n_{v}}}^{{ }_{n_{v}}^{n_{v}} p_{k}}\right) \\
=E\left\{{ }^{1} x^{1} p_{k} \times{ }^{2} x^{2} p_{k} \times \cdots \times{ }^{n_{v}} x{ }^{n_{v} p_{k}}\right\}, \forall\left({ }^{1} p_{k},{ }^{2} p_{k}, \cdots,{ }^{n_{v}} p_{k}\right) \in P
\end{aligned}
$$

O conjunto de potências aplicáveis é denominado $P=\left[\begin{array}{lllllll}P_{1} & P_{2} & \cdots & P_{k} & \cdots & P_{N_{E}}\end{array}\right]$, onde $P_{k}=\left[\begin{array}{lllllll}{ }^{1} p_{k} & { }^{2} p_{k} & \cdots & { }^{r} p_{k} & \cdots & { }^{n} p_{k}\end{array}\right]$ e ${ }^{r} p_{k}$ é o valor da $k$-ésima potência aplicada à $r$-ésima variável aleatória. O conjunto completo de equações é formado tomando-se todas as combinações de potências ao se tomar o conjunto $\left\{0,1, \cdots, O_{M}\right\}$, no qual $O_{M}$ é o maior momento das variáveis aleatórias a ser calculado, tal que ${ }^{1} p_{k}+{ }^{2} p_{k}+\cdots+{ }^{n_{v}} p_{k} \leq O_{M}$. Observando-se que o número de equações, $N_{E}$, deve ser maior ou igual ao número de incógnitas, $N_{I}$, pode-se aplicar um algoritmo iterativo incremental, no qual, partindo-se de um valor mínimo qualquer para $O_{M}$, adiciona-se uma unidade a este até que a inequação $N_{E}=$ $n_{v} ! /\left[n_{v} !-\left(O_{M}+1\right) !\right]-N_{r} \geq N_{I}$ seja satisfeita. Esta inequação fornece o número de permutações possíveis, observando-se que $N_{r}$ corresponde ao número de combinações com ordem equivalente maior do que $O_{M}$. Assim sendo, excluem-se as combinações que não satisfazem a restrição ${ }^{1} p_{k}+{ }^{2} p_{k}+\cdots+{ }^{n_{v}} p_{k} \leq O_{M}$.

Nos casos em que as variáveis aleatórias de entrada sejam não-correlacionadas, o processo de obtenção dos pesos e coordenadas sigma pode ser simplificado. As equações de equivalência, Eq. (1), podem ser desacopladas quanto aos termos referentes à cada uma das variáveis aleatórias, como mostra a Eq. (2), resultado em $n_{v}$ conjuntos independentes de equações, na forma da Eq. (3).

$$
\begin{aligned}
& \sum_{j_{1}=1}^{n_{s}} \sum_{j_{2}=1}^{n_{s}} \cdots \sum_{j_{n_{v}}=1}^{n_{s}} w_{j_{1}, j_{2}, \cdots, j_{n_{v}}} \times\left({ }^{1} x_{j_{1}}{ }^{1} p_{k} \times{ }^{2} x_{j_{2}}^{2} p_{k} \times \cdots \times{ }^{n_{v}} x_{j_{n_{v}}}^{n_{v_{v}} p_{k}}\right)=E\left\{{ }^{1} x^{1} p_{k} \times{ }^{2} x^{2} p_{k} \times \cdots \times{ }^{n_{v}} x^{n_{v} p_{k}}\right\} \\
& =\left(\sum_{j_{1}=1}^{n_{s}}{ }^{1} w_{j_{1}} \times{ }^{1} x_{j_{1}}^{1} p_{k}\right) \times\left(\sum_{j_{2}=1}^{n_{s}}{ }^{2} w_{j_{2}} \times{ }^{2} x_{j_{2}}^{2} p_{k}\right) \times \cdots \times\left(\sum_{j_{n_{v}}=1}^{n_{s}}{ }^{n_{v}} w_{j_{n_{v}}} \times{ }^{n_{v}} x_{j_{n_{v}}}^{n_{v} p_{p_{k}}}\right) \\
& =E\left\{{ }^{1} x^{1} p_{k}\right\} \times E\left\{{ }^{2} x^{2} p_{k}\right\} \times \cdots \times E\left\{{ }^{n_{v}} x^{n^{n_{v}} p_{k}}\right\}, \forall\left({ }^{1} p_{k},{ }^{2} p_{k}, \cdots,{ }^{n_{v}} p_{k}\right) \in P \\
& { }^{r} F_{k}=\sum_{j_{r}=1}^{n_{s}}{ }^{r} w_{j_{r}} \times{ }^{r} x_{j_{r}}^{k}-E\left\{{ }^{r} x^{k}\right\} \\
& \forall k=0,1, \cdots, O_{m} \in P, \forall r=1,2, \cdots, n_{v}
\end{aligned}
$$

Nesta equação, o peso ${ }^{r} w_{j_{r}}$, corresponde ao peso associado à variável $r$, é uma parcela do produtório da Eq. (4), que fornece o peso no ponto sigma formado pelas $n_{v}$ coordenadas, sendo cada um destes, então, referente à $j_{r}$-ésima coordenada da $r$-ésima variável. 


$$
w_{j_{1}, j_{2}, \cdots, j_{n_{v}}}=\prod_{r=1}^{n_{v}} r_{j_{j_{r}}}
$$

Neste caso, o vetor de incógnitas é dado pela Eq. (5) e o vetor-erro é dado pela Eq. (6).

$$
\begin{gathered}
{ }^{r} U=\left[\begin{array}{c}
{ }^{r} w_{1} \\
{ }^{r} w_{2} \\
\vdots \\
{ }^{r} w_{n_{s}} \\
{ }^{r} x_{1} \\
{ }^{r} x_{2} \\
\vdots \\
{ }^{r} x_{n_{s}}
\end{array}\right] \\
{ }^{r} F=\left[\begin{array}{c}
{ }^{r} F_{0} \\
{ }^{r} F_{1} \\
\vdots \\
{ }^{r} F_{O_{m}}
\end{array}\right]
\end{gathered}
$$

O Jacobiano de ${ }^{r} F$ é dado pela Eq. (7).

$$
\begin{gathered}
J r_{F}=\left[\begin{array}{cccccccc}
\frac{\partial^{r} F_{0}}{\partial^{r} w_{1}} & \frac{\partial^{r} F_{0}}{\partial^{r} w_{2}} & \cdots & \frac{\partial^{r} F_{0}}{\partial^{r} w_{n_{s}}} & \frac{\partial^{r} F_{0}}{\partial^{r} x_{1}} & \frac{\partial^{r} F_{0}}{\partial^{r} x_{2}} & \cdots & \frac{\partial^{r} F_{0}}{\partial^{r} x_{n_{s}}} \\
\frac{\partial^{r} F_{1}}{\partial^{r} w_{1}} & \frac{\partial^{r} F_{1}}{\partial^{r} W_{2}} & \cdots & \frac{\partial^{r} F_{1}}{\partial^{r} w_{n_{s}}} & \frac{\partial^{r} F_{1}}{\partial^{r} x_{1}} & \frac{\partial^{r} F_{1}}{\partial^{r} x_{2}} & \cdots & \frac{\partial^{r} F_{1}}{\partial^{r} x_{n_{s}}} \\
\vdots & \vdots & \ddots & \vdots & \vdots & \vdots & \ddots & \vdots \\
\frac{\partial^{r} F_{O_{m}}}{\partial^{r} W_{1}} & \frac{\partial^{r} F_{O_{m}}}{\partial^{r} W_{2}} & \cdots & \frac{\partial^{r} F_{O_{m}}}{\partial^{r} w_{n_{s}}} & \frac{\partial^{r} F_{O_{m}}}{\partial^{r} x_{1}} & \frac{\partial^{r} F_{O_{m}}}{\partial^{r} x_{2}} & \cdots & \frac{\partial^{r} F_{O_{m}}}{\partial^{r} x_{n_{s}}}
\end{array}\right] \\
\frac{\partial^{r} F_{k}}{\partial^{r} w_{j_{r}}}={ }^{r} x_{j_{r}}^{k} \\
\frac{\partial^{r} F_{k}}{\partial^{r} x_{j_{r}}}=k \times{ }^{r} w_{j_{r}} \times{ }^{r} x_{j_{r}}^{\max (0, k-1)}
\end{gathered}
$$

A solução ${ }^{r} U$ que faz com que $\left\|{ }^{r} F\right\|^{2} \leq \epsilon$, sendo $\epsilon$ o erro máximo tolerado, fornece as coordenadas dos pontos sigma que são associadas à $r$-ésima variável, bem como as parcela respectivas dos pesos de tais pontos. Neste trabalho, para a determinação dos valores numéricos das coordenadas e dos pesos sigma adotou-se o método globalmente convergente de Newton associado a buscas em linha (line searches) e rastreamento inverso (backtracking), de forma a incrementar a capacidade de busca (Press et al., 2002).

Tipicamente, tem-se um maior grau de convergência aplicando-se tal sistematização, pois, à medida em que cresce o número de incógnitas e o número de funções-erro, degrada-se a capacidade de localização de um ponto ótimo por parte do método de otimização. Assim sendo, é preferível aplicar o método de otimização várias vezes para um pequeno número de variáveis do que aplicá-lo uma vez tomando-se uma grande quantidade de incógnitas, o que é pertinente, visto a possibilidade de desacoplamento mostrada.

Dado o que foi exposto, o algoritmo de otimização aplicado é:

1) Para cada variável aleatória de entrada:

a. Calculam-se os valores esperados, $E\{\cdot\}$, das funções-erro, Eq. (3), aplicando integração numérica (Gonnet, 2012), cujo argumento de entrada é a função densidade de probabilidade da variável aleatória.

b. Aleatoriamente, gera-se uma proposta inicial de solução.

c. Aplica-se o algoritmo de Newton globalmente convergente.

d. Em caso de convergência, $\left\|^{r} F\right\|^{2}$, salta-se para o passo 2), retorna-se para o passo b, senão segue-se para o passo 2).

2) Calculam-se os pesos dos pontos sigma, formados pelas combinações das coordenadas sigma determinadas no passo 1), aplicando-se a Eq. (4). 
Os pontos sigma, os quais representam adequadamente a variação estatística das variáveis aleatórias de entrada, podem ser utilizados para determinar uma, também adequada, aproximação da função de mapeamento. Para isto, constróise um sistema linear superdeterminado, cuja solução fornece os coeficientes para o polinômio de aproximação (Press $e t$ al., 2002). O polinômio de aproximação é dado pela Eq. (8).

$$
\begin{aligned}
y=g(x) \approx f(x)= & \sum_{\substack{i_{1}=0 \\
o_{p}}}^{o_{i_{2}=0}} \sum_{\substack{o_{p} \\
\forall i_{1}, i_{2}, \cdots, i_{n_{v}}: i_{1}+i_{2}+\cdots+i_{n_{v}} \leq O_{p}}}^{o_{p}} a_{i_{1}, i_{2}, \cdots, i_{n_{v}}} \times\left({ }^{1} x^{i_{1}} \times{ }^{2} x^{i_{2}} \times \cdots \times{ }^{n_{v}} x^{i_{n_{v}}}\right),
\end{aligned}
$$

O parâmetro que indica a ordem do polinômio, $O_{p}$, é obtido de forma semelhante àquela aplicada para se encontrarem as potências da equação de equivalência. Aplica-se um algoritmo iterativo incremental, no qual, partindo-se de um valor mínimo arbitrário para $O_{p}$, adiciona-se iterativamente uma unidade a este enquanto a inequação $N_{p}=$ $n_{v}$ ! $\left[n_{v} !-\left(O_{p}+1\right) !\right]-N_{r} \leq N_{s}$ for satisfeita. Esta inequação fornece o número de permutações possíveis, na qual $N_{r}$ corresponde ao número de combinações com ordem equivalente maior do que $O_{p}$. Assim sendo, excluem-se as combinações que não satisfazem a restrição $i_{1}+i_{2}+\cdots+i_{n_{v}} \leq O_{p}$.

Estima-se do $k$-ésimo momento de uma variável de saída ao se calcular $E\left\{g(x)^{k}\right\}, x=\left({ }^{1} x,{ }^{2} x, \cdots,{ }^{n_{v}} x\right)$. Sabe-se que $E\left\{g(x)^{k}\right\} \approx E\left\{f(x)^{k}\right\}$, visto que $g(x) \approx f(x)$. Pode-se observar que a $k$-ésima potência da Eq. (8) pode ser dada pela Eq. (9), na qual os coeficientes $b_{(\cdot)}$ são funções dos coeficientes $a_{(\cdot)}$ do polinômio de aproximação.

$$
\begin{gathered}
f(x)^{k}=\sum_{i_{1}=0}^{k \times o_{p}} \sum_{i_{2}=0}^{k \times o_{p}} \cdots \sum_{\substack{i_{n_{v}}=0 \\
\forall \times o_{p}}}^{k} b_{i_{1}, i_{2}, \cdots, i_{n_{v}}} \times\left({ }^{1} x^{i_{1}} \times{ }^{2} x^{i_{2}} \times \cdots \times{ }^{n_{v}} x^{i_{n_{v}}}\right) \\
\forall i_{1}, i_{2}, \cdots, i_{n_{v}}: i_{1}+i_{2}+\cdots+i_{n_{v}} \leq k \times o_{p}
\end{gathered}
$$

Assim, pode-se reescrever a Eq. (9) em termos de $E\left\{f(x)^{k}\right\}$, como mostra a Eq. (10).

$$
\begin{aligned}
& E\left\{f(x)^{k}\right\}=\sum_{i_{1}=0}^{k \times o_{p}} \sum_{i_{2}=0}^{k \times o_{p}} \cdots \sum_{i_{n_{v}}=0}^{k \times o_{p}} b_{i_{1}, i_{2}, \cdots, i_{n_{v}}} \times E\left\{{ }^{1} x^{i_{1}} \times{ }^{2} x^{i_{2}} \times \cdots \times{ }^{n_{v}} x^{i_{n_{v}}}\right\} \\
& \forall i_{1}, i_{2}, \cdots, i_{n_{v}}: i_{1}+i_{2}+\cdots+i_{n_{v}} \leq k \times O_{p}
\end{aligned}
$$

Tomando-se a Eq. (1), pode-se reescrever a Eq. (10) na forma da Eq. (11).

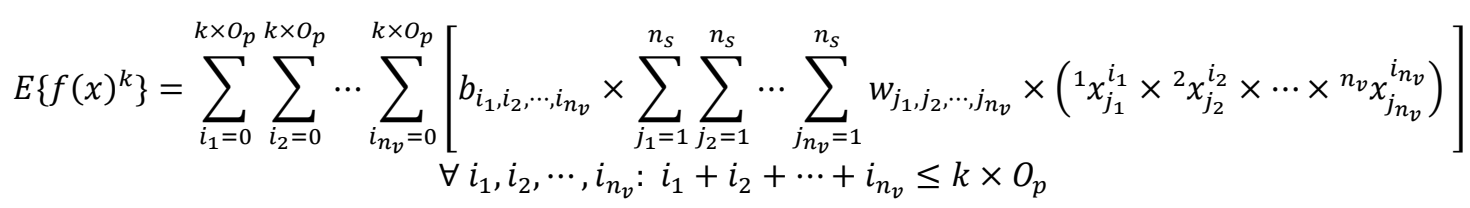

A ordem dos somatórios da Eq. (11) pode ser invertida, de forma que resulta na Eq. (12).

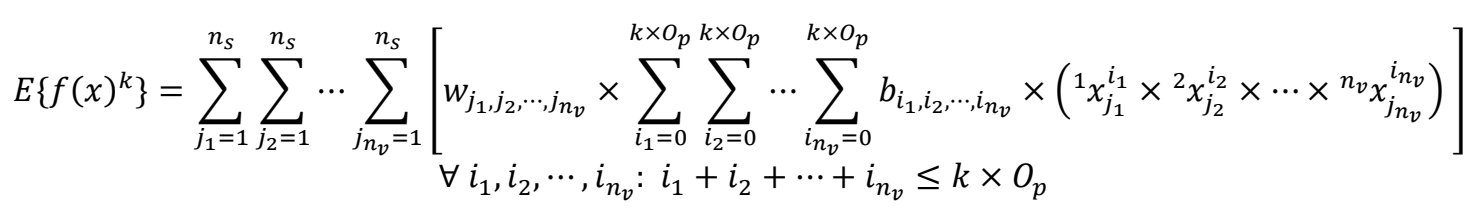

Resta, então, observar que os somatórios internos da Eq. (12) correspondem às avaliações de $f(x)^{k}$ nos pontos sigma, na mesma forma da Eq. (8). Estas podem ser precisamente representadas pela função original de mapeamento $g(x)$, de forma que a expressão final se torna a Eq. (13).

$$
E\left\{g(x)^{k}\right\} \approx \sum_{j_{1}=1}^{n_{s}} \sum_{j_{2}=1}^{n_{s}} \ldots \sum_{j_{n_{v}}=1}^{n_{s}} w_{j_{1}, j_{2}, \cdots, j_{n_{v}}} \times\left[g\left({ }^{1} x_{j_{1}},{ }^{2} x_{j_{2}}, \cdots,{ }^{n_{v}} x_{j_{n_{v}}}\right)\right]^{k}
$$


Portanto, após a determinação dos valores ótimos dos pontos sigma e de seus pesos, pode-se estimar diretamente os momentos centrais das funções de mapeamento. Para isto, aplicam-se as Eqs. (14), derivadas da Eq. (13), tomando-se a função de mapeamento $y=g(x)$.

$$
\begin{aligned}
& \bar{y} \approx \sum_{j_{1}=1}^{n_{s}} \sum_{j_{2}=1}^{n_{s}} \ldots \sum_{j_{n_{v}}=1}^{n_{s}} w_{j_{1}, j_{2}, \cdots, j_{n_{v}}} \times g\left({ }^{1} x_{j_{1}},{ }^{2} x_{j_{2}}, \cdots,{ }^{n_{v}} x_{j_{n_{v}}}\right) \\
& \sigma^{2} \approx \sum_{j_{1}=1}^{n_{s}} \sum_{j_{2}=1}^{n_{s}} \ldots \sum_{j_{n_{v}}=1}^{n_{s}} w_{j_{1}, j_{2}, \cdots, j_{n_{v}}} \times\left[g\left({ }^{1} x_{j_{1}},{ }^{2} x_{j_{2}}, \cdots,{ }^{n_{v}} x_{j_{n_{v}}}\right)-\bar{y}\right]^{2} \\
& \gamma_{1} \sigma^{3} \approx \sum_{j_{1}=1}^{n_{s}} \sum_{j_{2}=1}^{n_{s}} \ldots \sum_{j_{n_{v}}=1}^{n_{s}} w_{j_{1}, j_{2}, \cdots, j_{n_{v}}} \times\left[g\left({ }^{1} x_{j_{1}},{ }^{2} x_{j_{2}}, \cdots,{ }^{n_{v}} x_{j_{n_{v}}}\right)-\bar{y}\right]^{3} \\
& \gamma_{2} \sigma^{4} \approx \sum_{j_{1}=1}^{n_{s}} \sum_{j_{2}=1}^{n_{s}} \ldots \sum_{j_{n_{v}}=1}^{n_{s}} w_{j_{1}, j_{2}, \cdots, j_{n_{v}}} \times\left[g\left({ }^{1} x_{j_{1}},{ }^{2} x_{j_{2}}, \cdots,{ }^{n_{v}} x_{j_{n_{v}}}\right)-\bar{y}\right]^{4}
\end{aligned}
$$

Cabe ressaltar que o termo constante $\bar{y}$ pode ser incluído na Eq. (8) simplesmente assumindo a sua composição com o termo $a_{0,0, \cdots, 0}$ do somatório, também constante, sem que se perca a generalização para os momentos centrais que são explicitados nas Eqs. (14).

\section{APLICAÇÃO}

Como exemplo ilustrativo, mostra-se na Fig. 1, o resultado da Transformada da Incerteza de uma variável aleatória gaussiana com média nula e desvio-padrão igual a um, tomando-se 5 (cinco) pontos sigma. A transformação corresponde a encontrar os pontos sigma da variável aleatória e seus respectivos pesos solucionando-se a Eq. (1), de forma que, para o propósito de cálculo dos momentos centrais, a avaliação massiva de uma distribuição contínua pode ser substituída pela avaliação de uma função de mapeamento em poucos pontos.

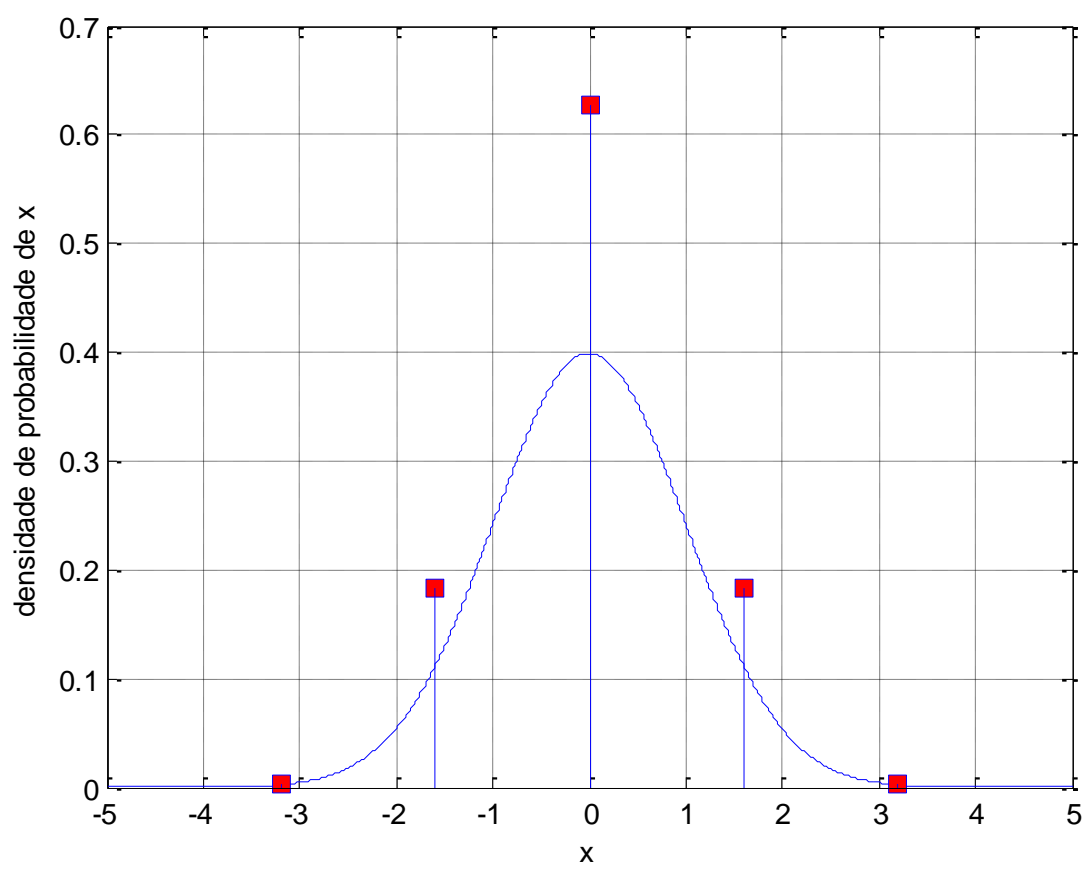

Figura 1. Ilustração da equivalência entre distribuição contínua e distribuição discreta de probabilidade.

Como exemplo de aplicação real, pretende-se estimar os momentos centrais da impedância de entrada de uma antena dipolo, dadas as incertezas quanto às dimensões da antena, realizando-se um pequena quantidade de simulações do modelo numérico da antena. Modelam-se tanto o diâmetro quanto o comprimento da antena por meio de distribuições gaussianas. O diâmetro possui um valor nominal de 0.005 de um comprimento de onda e o comprimento apresenta um valor nominal de 0.5 de um comprimento de onda. A incerteza é modelada, no primeiro caso, considerando-se $2.5 \%$ de desvio-padrão referentes aos valores nominais de comprimento e de diâmetro. No segundo caso, é considerado um desvio 
de 5\%. Foi utilizado um simulador numérico de antenas que emprega o Método dos Elementos Finitos (Menezes et al., 2010).

A Fig. 2 mostra as localizações e pesos dos pontos sigma em verde, tomando-se 4 pontos por variável aleatória, totalizando-se 16 avaliações do modelo numérico da antena. A Fig. 3 mostra o mapeamento entrada-saída, na qual, à esquerda, têm-se as variáveis aleatórias de entrada, diâmetro (eixo horizontal) e comprimento (eixo vertical) da antena, e, à direita, têm-se as saídas, parte real (eixo horizontal) e parte imaginária (eixo vertical) da impedância da antena. Em ambas as figuras, tomam-se desvios relativos iguais a $2.5 \%$

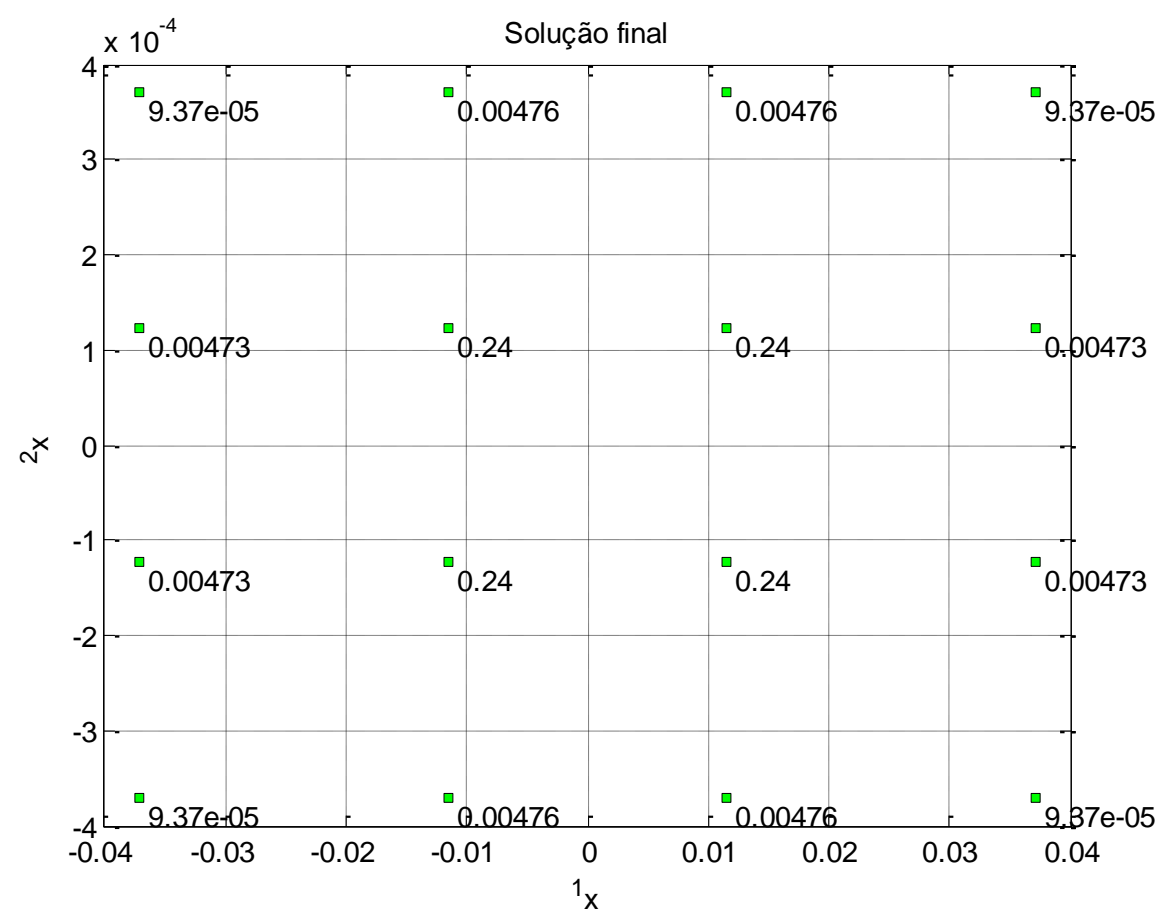

Figura 2. Pontos sigma e pesos para $n_{s}=4$ e desvios de $2.5 \%$ referentes aos valores nominais.
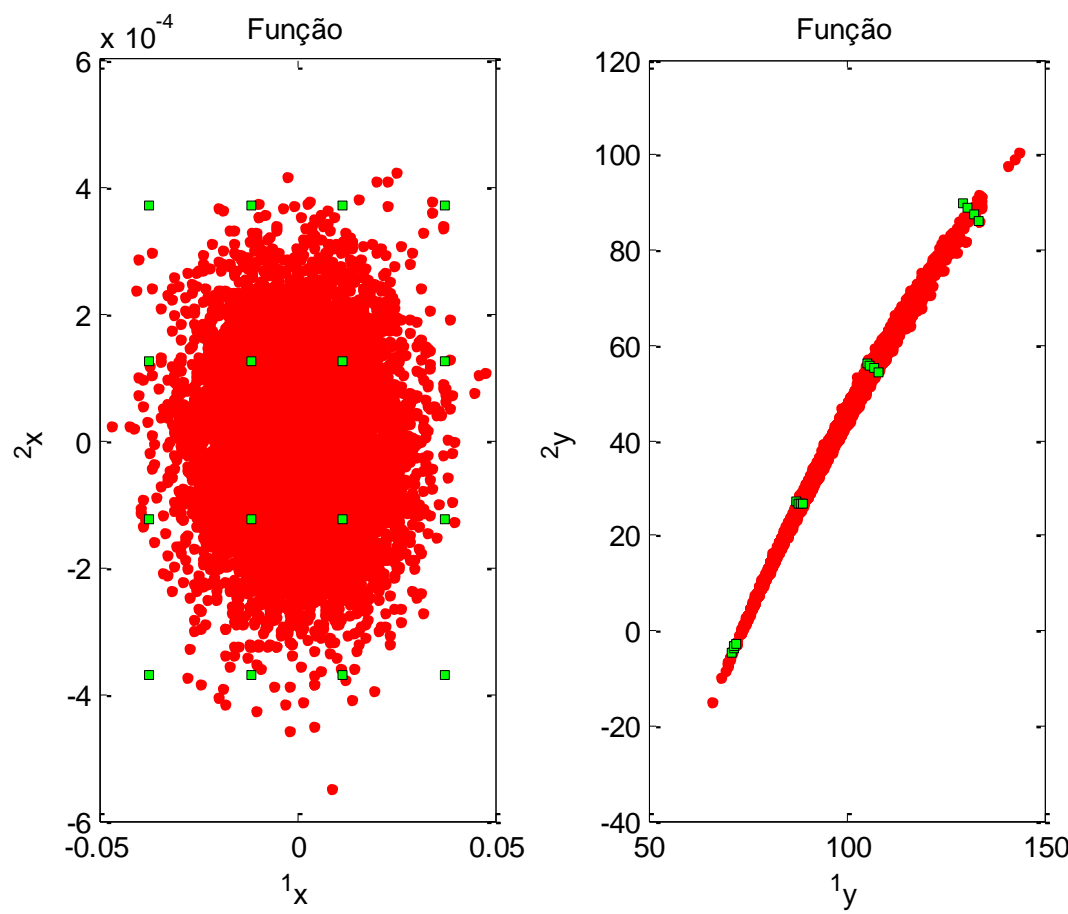

Figura 3. Mapeamento entradas-saídas para $n_{s}=4$ e desvios de $2.5 \%$ referentes aos valores nominais. Os pontos em vermelho correspondem às amostras aleatórias das variáveis de entrada, à direita, e seus respectivos mapeamentos, à esquerda. 
A Tab. 1, bem como as demais, mostra os resultados obtidos ao se aplicar a Eq. (14) para o cálculo dos momentos centrais pela Transformada da Incerteza. São mostrados também, para o propósito de observação da precisão, os momentos calculados a partir da avaliação de uma massa de $10^{4}$ amostras da função de mapeamento.

Tabela 1. Momentos centrais obtidos pela Transformada da Incerteza.

\begin{tabular}{|c|c|c|c|c|c|}
\hline Desvio-padrão & & Média & Variância & Assimetria & Curtose \\
\hline $2.5 \%$ & ${ }^{1} y$ & 97.5502 & 100.0730 & 0.1927 & 2.4276 \\
\cline { 2 - 6 } & ${ }^{2} y$ & 41.1113 & 239.1871 & 0.0337 & 2.1987 \\
\hline \multirow{2}{*}{$\begin{array}{c}10^{4} \text { avaliações } \\
\text { das funções }\end{array}$} & ${ }^{1} y$ & 97.5958 & 102.4209 & 0.3007 & 3.1124 \\
\cline { 2 - 6 } & ${ }^{2} y$ & 41.1695 & 243.5186 & 0.0466 & 2.9331 \\
\hline
\end{tabular}

As Figs. 4 e 5 apresentam os resultados para desvios relativos iguais a 5.0\%.

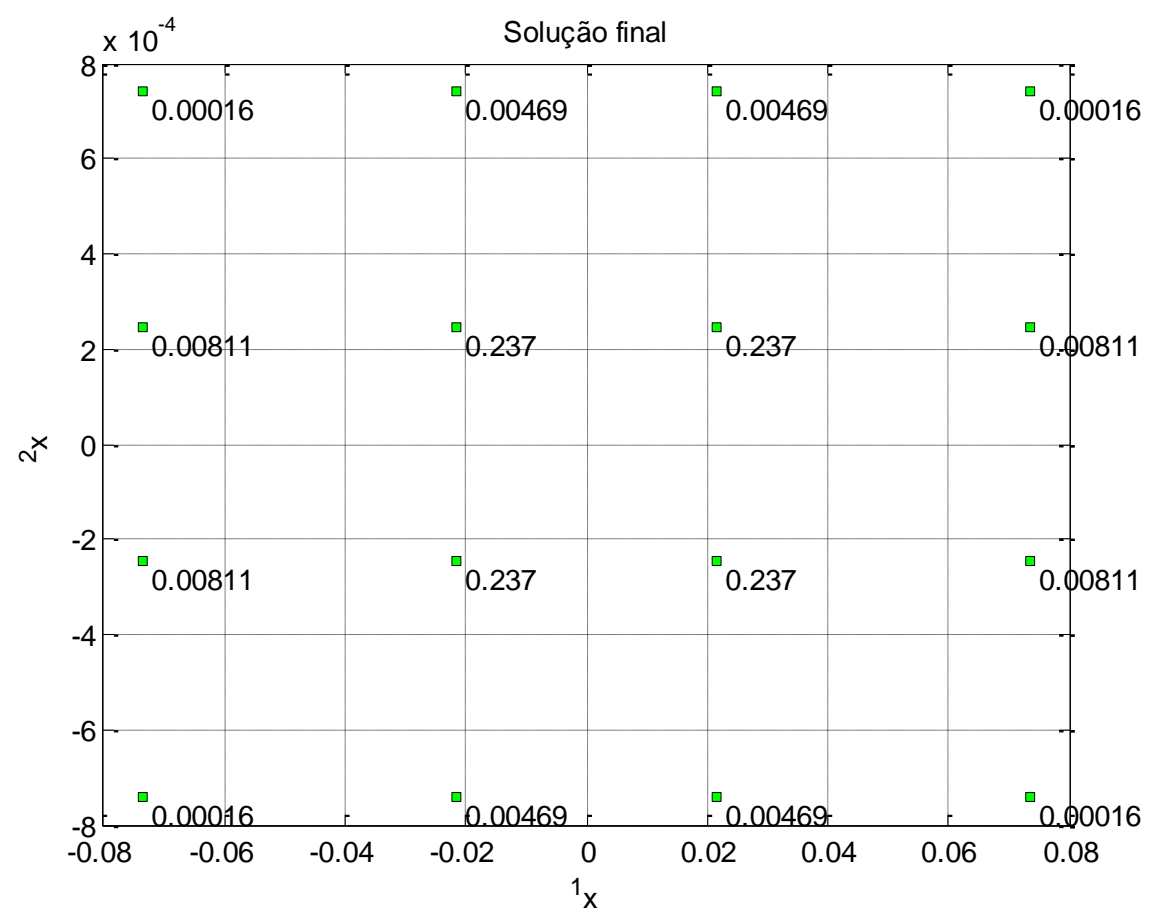

Figura 4. Pontos sigma e pesos para $n_{s}=4$ e desvios de $5 \%$ referentes aos valores nominais. 

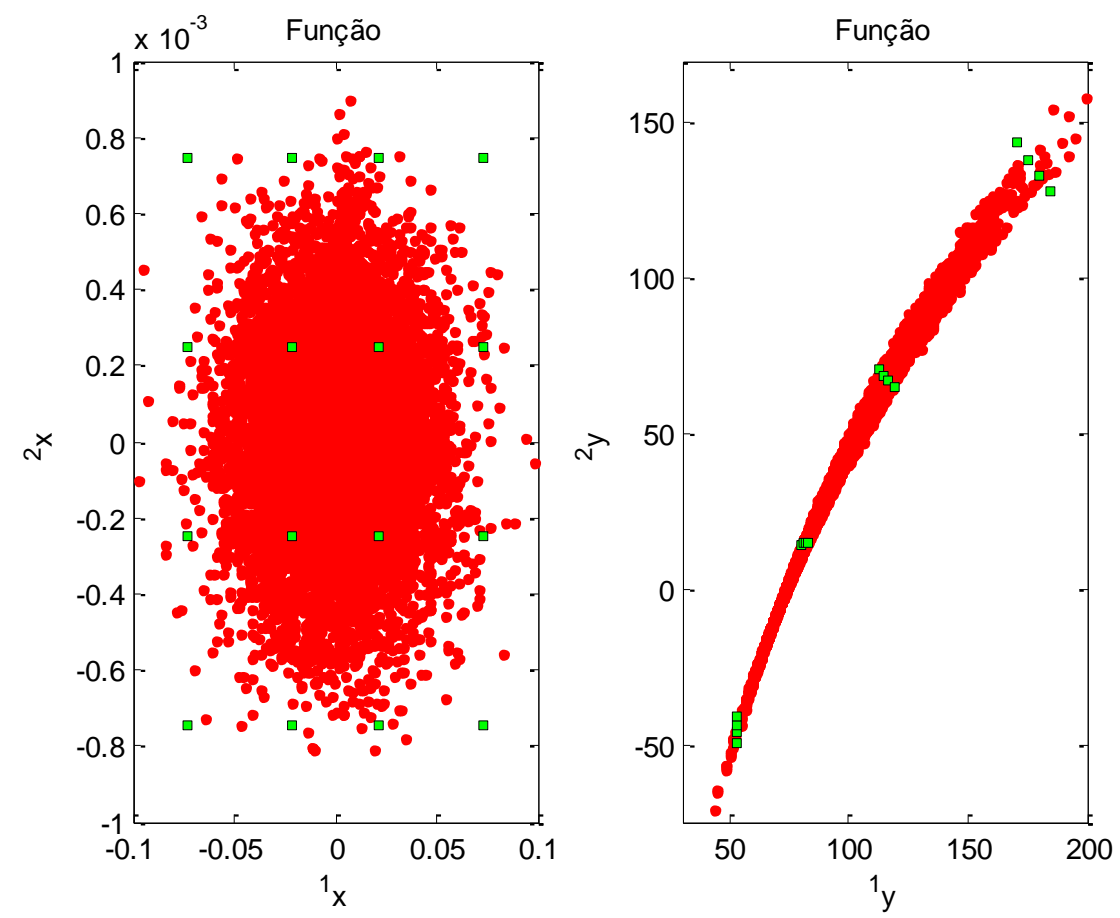

Figura 5. Mapeamento entradas-saídas, para $n_{s}=4$ e desvios de $5 \%$ referentes aos valores nominais.

Tabela 2. Momentos centrais obtidos pela Transformada da Incerteza.

\begin{tabular}{|c|c|c|c|c|c|}
\hline Desvio-padrão & & Média & Variância & Assimetria & Curtose \\
\hline \multirow{2}{*}{$5.0 \%$} & ${ }^{1} y$ & 99.1057 & 424.2734 & 0.6862 & 4.4409 \\
\cline { 2 - 6 } & ${ }^{2} y$ & 41.5147 & 954.1666 & 0.1017 & 2.9904 \\
\hline \multirow{2}{*}{$\begin{array}{c}0^{4} \text { avaliações } \\
\text { das funções }\end{array}$} & ${ }^{1} y$ & 98.7287 & 411.8545 & 0.5842 & 3.5841 \\
\cline { 2 - 6 } & ${ }^{2} y$ & 41.0096 & 941.9281 & 0.0681 & 2.9755 \\
\hline
\end{tabular}

Finalmente, a Tab. 3 mostra, tomando-se 15 coordenadas sigma por variável aleatória de entrada, o resultados referentes aos momentos centrais das funções de mapeamento.

Tabela 3. Momentos centrais obtidos pela Transformada da Incerteza.

\begin{tabular}{|c|c|c|c|c|c|}
\hline Desvio-padrão & & Média & Variância & Assimetria & Curtose \\
\hline \multirow{2}{*}{$2.5 \%$} & ${ }^{1} y$ & 97.5505 & 100.5614 & 0.3106 & 3.1767 \\
\cline { 2 - 6 } & ${ }^{2} y$ & 41.1116 & 239.1323 & 0.0562 & 3.0010 \\
\hline \multirow{2}{*}{$\begin{array}{c}10^{4} \text { avaliações } \\
\text { das funções }\end{array}$} & ${ }^{1} y$ & 97.4049 & 99.7418 & 0.2911 & 3.1056 \\
\cline { 2 - 6 } & ${ }^{2} y$ & 40.8890 & 238.1626 & 0.0403 & 2.9742 \\
\hline
\end{tabular}

Pode-se observar nas Tabelas 1, 2 e 3 que a Transformada da Incerteza aproxima com razoável precisão os momentos centrais obtidos por meio de avaliações massivas das funções de mapeamento ( $10^{4}$ avaliações das funções de mapeamento). Tal precisão, obviamente, é maior quanto maior for o número de coordenadas sigma consideradas.

\section{CONCLUSÃO}

Neste trabalho, foi apresentada a formulação que permite a solução numérica das equações da Transformada da Incerteza, de forma que sua aplicação ágil se torna factível. O método trata de quaisquer distribuições de probabilidade, com precisão dependente do número de coordenadas sigma pré-definidas, e de quaisquer funções de mapeamento. Obtêmse, como resultado, os momentos centrais das respostas das funções de mapeamento, o que é de grande utilidade nos processos de avaliação de incertezas em problemas com avaliações moderada ou altamente custosas, visto que o método de Monte Carlo pode ser inviável devido à demanda por uma quantidade massiva de avaliações. A abordagem proposta foi testada em vários problemas analíticos e numéricos de Engenharia e todos os resultados se mostraram satisfatórios.

A abordagem proposta pode ser prontamente aplicada à um método de otimização robusta que avalie as incertezas inerentes às variáveis de projeto a serem otimamente dimensionadas. 


\title{
5. AGRADECIMENTOS
}

Os autores agradecem à Capes/MEC e à FAPEG (Fundação de Amparo à Pesquisa do Estado de Goiás).

\section{REFERÊNCIAS}

Borges, G., Júnior, E., \& Menezes, L. (2008). Algorithms for uncertainty propagation in transmission-line matrix (TLM) method. International Journal of Numerical Modelling, 21, pp. 43-60.

Cacuci, D. (2003). Sensitivity and Uncertainty Analysis - Vol. I, Theory. NY: CRC Press.

Gonnet, P. (2012). A review of error estimation in adaptive quadrature. ACM Computing Surveys, 44(4).

Ishihara, J. Y., Silva, L. M., Soares, A. J., \& Menezes, L. R. (2013). Using unscented transform as alternative to Monte Carlo in bit error rate calculations. Electronics Letters, 49, pp. 675-677.

Julier, S., \& Uhlmann, J. (2004). Unscented Filtering and Nonlinear Estimation. Proceedings of the IEEE, 92(3), pp. 401422.

Menezes, L., Ajayi, A., Christopoulos, C., Sewell, P., \& Borges, G. (2007). Efficient computation of stochastic electromagnetic problems using unscented transforms. IET - Science, Measurement \& Technology, 2, pp. 88-95.

Menezes, L., Soares, A., Silva, F., Terada, M., \& Correia, M. (2010). A New Procedure for Assessing the Sensitivity of Antennas Using the Unscented Transform. IEEE Transactions on Antennas and Propagation, 58, pp. 988-993.

Paredes, A., Menezes, L., \& Abdalla, H. (2011). Statistical Modeling of Manufacturing Uncertainties for Microstrip Filters. Journal of Microwaves, Optoelectronics and Electromagnetic Applications, 10, pp. 179-202.

Paredes, A., Menezes, L., Abdalla, H., \& Romani, I. (2010). Probabilistic finite element analysis of radiofrequency liver ablation using the unscented transform. Modelling and Simulation in Engineering, 2010, pp. 1-5.

Press, H., Teukolsky, S., Vetterling, W., \& Flannery, B. (2002). Numerical Recipes in C. NY: Cambridge University Press.

Santos, I., Haemmerich, D., Schutt, D., Rocha, A., \& Menezes, L. (2009). Probabilistic finite element analysis of radiofrequency liver ablation using the unscented transform. Physics in Medicine and Biology, 54, pp. 627-640.

Thomas, D., Menezes, L., Christopoulos, C., Leferink, F., \& Bergsma, H. (2011). Estimation of the probability distributions for cable coupling using Unscented Transforms. Annales des Télécommunications, 66, pp. 475-482.

\section{RESPONSABILIDADE AUTORAL}

Os autores são os únicos responsáveis pelo conteúdo deste trabalho.

\section{FULL NUMERICAL UNSCENTED TRANSFORM FOR MAPPING OF PROBABILITY DISTRIBUTIONS}

\author{
Leonardo da Cunha Brito, brito@emc.ufg.br ${ }^{1}$ \\ Leonardo Rodrigues Araújo Xavier de Menezes, leonardo@ene.unb.br ${ }^{2}$ \\ Adson Silva Rocha, adson.rocha@ifgoiano.edu.br ${ }^{3}$ \\ Ademir Alves de Brito Junior, ademir_britojr@hotmail.com ${ }^{1}$
}

${ }^{1}$ Universidade Federal de Goiás - Escola de Engenharia Elétrica, Mecânica e de Computaão, Av. Universitária, n. ${ }^{\circ}$ 1488, qd. 86, bloco A, $3^{\circ}$ piso, Setor Leste Universitário, CEP 74605-010, Goiânia/GO

${ }^{2}$ Universidade de Brasília - Departamento de Engenharia Elétrica, CEP 70900-900, Brasília/DF

${ }^{3}$ Instituto Federal Goiano - Rua 88, n 310, Setor Sul, CEP 74085-010, Goiânia/GO

\begin{abstract}
In this work, we developed a numerical version of the Unscented Transform. In the approach developed, any probability distributions can be mapped by linear or non-linear functions, thus enabling fast acquisition of the probability distributions of the outputs of the simulation model, or more specifically, the evaluation of model uncertainties. An appropriate degree of accuracy can be achieved, since the equation allows the arbitrary increment of sample points of mapping functions, at the expense of increased computational cost. Nevertheless, for practical purposes of mapping distributions, the computational cost is still considerably lower than that demanded, for example, by the Monte Carlo method, which is based on a massive random sampling, thus presenting a very high computational cost. Applications in problems of assessment of uncertainties in engineering show the applicability and efficiency of the proposed method.
\end{abstract}

Keywords: mapping, probability distribution, Unscented Transform, numerical version 